\title{
Décadrages Décadrages
}

cinéma, à travers champs Cinéma, à travers champs

$18 \mid 2011$

Mario Ruspoli et le « cinéma direct »

\section{Compte rendu}

\section{Hélène Fleckinger (éd.), Carole Roussopoulos : Caméra} militante. Luttes de libération des années 1970

Genève, Editions MētisPresses, 2010

\section{Sylvain Portmann}

\section{OpenEdition}

\section{Journals}

Édition électronique

URL : https://journals.openedition.org/decadrages/234

DOI : $10.4000 /$ decadrages. 234

ISSN : 2297-5977

\section{Éditeur}

Association Décadrages

\section{Édition imprimée}

Date de publication : 10 avril 2011

Pagination : 137-139

ISBN : 978-2-9700668-2-8

ISSN : 2235-7823

Référence électronique

Sylvain Portmann, « Hélène Fleckinger (éd.), Carole Roussopoulos : Caméra militante. Luttes de libération des années $1970 »$, Décadrages [En ligne], 18 | 2011, mis en ligne le 10 avril 2012, consulté le 03 avril 2022. URL : http://journals.openedition.org/decadrages/234 ; DOI : https://doi.org/10.4000/ decadrages. 234 
par Sylvain Portmann

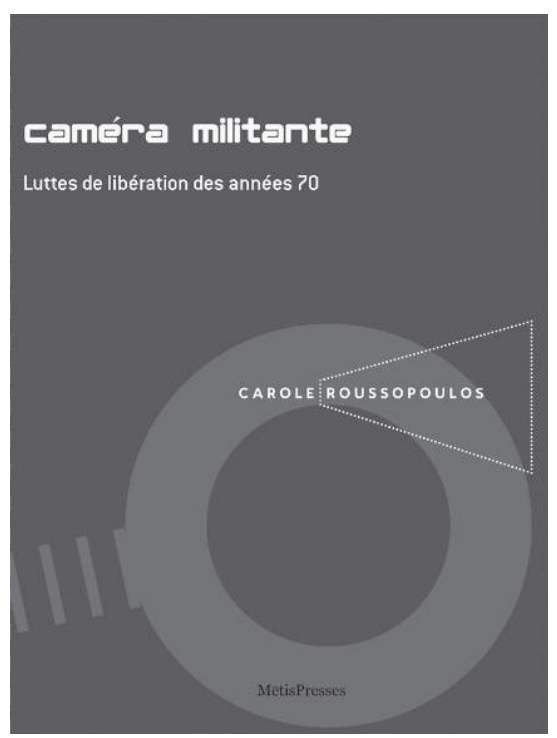

Hélène Fleckinger (éd.), Carole Roussopoulos: Caméra militante. Luttes de libération des années 1970, Genève, Editions MētisPresses, 2010, 131 p. + 1 DVD

Deuxième titre de la collection PLANSécant, l'ouvrage est composé d'une série d'articles consacrés à Carole Roussopoulos et d'un DVD rassemblant six de ses vidéos, datant de 1970 à 19761. Les textes, tout comme un entretien de la réalisatrice mené en 2007, fournissent ici des informations et des analyses essentielles pour approcher l'œuvre de la vidéaste, décédée en septembre 2009. Les éditions MētisPresses présentent avec ce volume la première monographie consacrée exclusivement à Carole Roussopoulos, qui a pourtant signé plus d'une centaine de documentaires, mais dont la place, en bibliothèque en tout cas, n'était pas encore évaluée à sa juste valeur. Véritable pionnière dans le
1 Les vidéos sont signées Carole Roussopoulos ou du nom de collectifs tels que Vidéo Out ou Les Insoumuses. Le DVD contient: Genet parle d'Angela Davis (1970), Le F.H.A.R. (Front Homosexuel d'Action Révolutionnaire, 1971), Monique (Lip I, 1973), Christiane et Monique (Lip V, 1976), S.C.U.M. Manifesto (1976), Maso et et Miso vont en bateau (1976). 
$2 \mathrm{~A}$ ce sujet, voir l'importance que prend C. Roussopoulos au sein de Vidéo, la mémoire au poing d'Anne-Marie Duguet (Paris, Hachette, 1981) Iorsqu'elle traite de l'arrivée de la vidéo en France ou de son investissement concret (initiation aux techniques de la vidéo par exemple) au profit de combats politiques menés en France et hors d'Europe.

3 L'apparition de la vidéaste dans l'index de Vidéo: un art contemporain de Françoise Parfait (Paris, Editions du Regard, 2001) est éclairant: son nom renvoie à une vignette (p. 191), ou plus exactement à la captation avec Gérard Cairashi d'une performance réalisée par Michel Journiac intitulée Messe pour un corps en 1975, et dont il est alors question dans l'ouvrage. Mais son nom ne fait que légender l'image. Dans L'art vidéo de Michael Rush (Paris, Thames \& Hudson, 2003), son nom n'apparaît pas.

4 Une filmographie détaillée est disponible sur le site www.carole-roussopoulos.com. champ de la vidéo engagée des années 1970, elle a incarné un système de production indépendant, s'immisçant au cœur de nombreux conflits sociaux au moment où ils eurent lieu 2 . Signalons aussi son absence répétée dans nombre d'ouvrages portant sur l'histoire de la vidéo, écartée peut-être pour le maintien d'une certaine conception de l'art vidéo ${ }^{3}$.

Quatre critiques ont participé à l'ouvrage : Nicole Brenez, Jean-Paul Fargier, Hélène Fleckinger (à qui l'on doit la sélection des films et l'édition des textes) et François Bovier (directeur avec Hamid Taieb de la collection PLANSécant). Ils livrent là un excellent point de départ à celui ou celle qui désire aborder une ouvre qui n'a cessé de «donner la parole» aux femmes, aux homosexuel(le)s, aux sans-abris, aux toxicomanes, aux personnes âgées. La liste est longue, tout comme celle de ses films 4 . Ceux proposés sur le DVD remontent à la période des six premières années de réalisation vidéo de cette Valaisanne d'origine, alors installée à Paris. Le choix se porte ainsi sur le début de l'œuvre, laissant à Carole Roussopoulos, à travers l'entretien qui clôt le livret, le soin d'aborder plus globalement sa biographie et ses projets ultérieurs.

Genet parle d'Angela Davis est la première vidéo de Carole Roussopoulos et la seule trace audiovisuelle de cette lecture par Jean Genet d'une lettre initialement prévue pour une diffusion à la télévision française, en 1970. Genet en personne l'avait incitée peu auparavant à s'équiper de matériel vidéo et c'est lui qui l'avait sollicitée afin de «seconder» l'équipe sur place, craignant que son intervention ne fut censurée. $\mathrm{Ce}$ que l'ORTF ne manqua pas de faire. C'est donc une voix (celle, certes, d'un personnage public) et le dispositif télévisuel alentour qui sont captés. On peut déjà y déceler une thématique essentielle à l'œuvre qui va suivre, celle de la voix et de sa diffusion. Jean-Paul Fargier propose une lecture détaillée de la vidéo tout en formulant quelques principes et objectifs qui selon lui définissent la vidéo militante. Il expose comment la longueur des plans, les mouvements de l'appareil, ou la choralité des discours enregistrés sont au service d'une diffusion large tout en s'opposant violemment à la télévision. Ajoutons à son analyse que si c'est la voix de Genet que l'on entend (et son visage qu'on voit), ce dernier compte bien en faire entendre une autre, celle d'Angela Davis, alors emprisonnée aux Etats-Unis, elle-même porte-parole des Black Panthers.

Ce schéma d'imbrication (ou de relais) revient fréquemment dans les bandes de Carole Roussopoulos. François Bovier, dont l'analyse richement documentée du mouvement «LIP» permet une vision détaillée des différentes composantes du conflit, signale ce processus d'intrication de paroles dans le film Monique (Lip 1). Dans ce cas, c'est la voix des femmes au sein du mouvement ouvrier qui est au centre de la bande 
vidéo : la révolte y est organisée autour d'un Comité d'Action, chapeautant des commissions, regroupant elles-mêmes des sous-commissions. Et à cela se mêle la participation des syndicats CFDT et CGT. Si le mouvement de protestation général s'adresse aux "patrons", certaines voix remettent en question son fonctionnement interne qui peine à prendre en compte les revendications féministes. A ce sujet, Hélène Fleckinger rappelle dans son intervention que "l'ouvrier est sexué» $\mathbf{5}$ et que les mouvements syndicaux sont bien moins homogènes que certains ne l'imaginent. Monique approche la situation avec un point de vue précis, celui d'une ouvrière qui témoigne des forces en jeu, avec un discours aussi clair que franc. C'est d'ailleurs cette même personne qui affirme qu'à la télévision, "on tronque, on coupe les phrases et ça ne ressemble plus à ce que ça veut dire» $\mathbf{6}$. Jean-Paul Fargier et Hélène Fleckinger insistent tous deux sur le combat permanent que Carole Roussopoulos a mené contre la télévision, ou à côté de la télévision, mettant toujours un point d'honneur à produire et maîtriser la diffusion de ses films.

Si le titre de l'ouvrage annonce une «caméra militante» et des «luttes de libération", les lecteurs ou spectateurs peu connaisseurs de l'œuvre de Carole Roussopoulos seront étonnés par l'humour qui traverse toutes ses œuvres, même lorsque les sujets sont des plus délicats: il est possible chez elle de rire tout en dénonçant la pratique des mutilations sexuelles $\mathbf{7}$, pour ne citer qu'un exemple. Nicole Brenez nous prévenait - à la première phrase du livret déjà - que c'était "avec une énergie inépuisable, un humour ravageur, une pertinence historique $\mathbf{8}$ que l'artiste travaillait. L'humour se donne même comme un élément constitutif de son idée de l'engagement: "Le mouvement de libération des femmes, qui a duré à mon avis très peu de temps, était vraiment lié à cette subversion et à cet humour. C'est comme ça qu'on peut gagner des luttes, ce n'est pas en faisant du militantisme ennuyeux où on se sacrifie dans des réunions» 9 .

Et pour ceux qui désirent poursuivre la (re)découverte des œuvres de Carole Roussopoulos, signalons que ses archives ont été déposées à la Médiathèque du Valais : "C'était une occasion inespérée d'avoir de l'aide pour ranger mes archives. [...] Chaque producteur va recevoir maintenant un courrier leur disant où sont les films. Ils pourront y avoir accès et je suis extrêmement contente»10. Les spectateurs intéressés sont désormais avertis.
5 Hélène Fleckinger, "Une caméra à soi. Quand les féministes s'emparent de la vidéo ", Carole Roussopoulos: Caméra militante. Luttes de libération des années 1970, op. cit., p. 41.

6 Monique (Lip I), (Vidéo Out, Carole et Paul Roussopoulos, France, 1973).

7 Voir Femmes mutilées, plus jamais (Fatxiya Ali Aden, Sahra Osman, avec la collaboration de Carole Roussopoulos, Suisse, 2007).

8 Nicole Brenez, "Carole Roussopoulos ou (')attention créatrice`", Carole Roussopoulos: Caméra militante. Luttes de libération des années 1970, op. cit., p. 7.

9 Hélène Fleckinger, "Marcher le vent au nez. Entretien avec Carole Roussopoulos", Carole Roussopoulos: Caméra militante. Luttes de libération des années 1970, op. cit., p. 107.

10 Extrait de l'émission radiophonique Temps d'arrêt, 25 février 2008 (prod. Nicole Michlig), Rhône FM. Disponible à l'écoute sur le site de la médiathèque Valais-Martigny (http://archives. memovs.ch). 